\title{
SALUD PÚBLICA Y LOS DERECHOS HUMANOS DE LOS ADULTOS MAYORES
}

\author{
Martha B. Peláez \\ Marcela Ferrer Lues**
}

\section{Resumen}

Destacando que la necesidad de proteger los derechos humanos de los adultos mayores no ha sido reconocida por la comunidad internacional, el trabajo analiza la discriminación en razón de la edad que pueden sufrir los adultos mayores. Se abordan las dimensiones de salud, laboral y de las transferencias intergeneracionales como las tres áreas donde puede apreciarse tal discriminación, distinguiendo la vulnerabilidad en razón de la edad, de la vulnerabilidad que obedece a otros factores, tales como el género, la raza, la etnia o el estrato socioeconómico. La discriminación por edad se expresa en un conjunto de acciones $\mathrm{u}$ omisiones que tienden a tratar al individuo o a una colectividad con estándares inferiores en razón exclusiva de la edad. Se muestra que la discriminación por edad no actúa aislada, sino que actúa conjuntamente con otros factores, aumentado el riesgo de vulnerabilidad y potenciando la discriminación. Es el caso, por ejemplo, de la salud, el género y las desigualdades económicas asociadas a la discriminación por edad. Para concluir, se sugiere una serie de lineamientos de políticas públicas orientadas a reducir las iniquidades relacionadas con la edad y construir una sociedad justa

$\mathrm{Ph}$. D. en Filosofía, Universidad de Tulane, New Orleans, USA. Asesora Regional en Envejecimiento y Salud, Programa de la Salud de la Familia y la Población, División de Promoción y Protección a la Salud, OPS/OMS, Washington, D.C.

Correspondencia: pelaezma@paho.org

** Investigadora del Centro de Análisis de Políticas Públicas de la Universidad de Chile.

Correspondencia: marcela.ferrerlues@utoronto.ca

\section{Abstract \\ Public health and the human rights of the elderly}

By underlying the fact that the need to protect the human rights of the elderly has not yet been recognized by the international community, this paper analyses the discrimination older people may suffer because of age. Health, work and intergenerational transferences are addressed as the three areas where such a discrimination may be perceived; we distinguish vulnerability by reason of age from vulnerability due to other factors, such as gender, race, ethnic minority or socioeconomic status. Discrimination by reason of age is expressed through a number of actions or omissions aimed at treating individuals or collectivities under lower standards only because of age. It is evident that discrimination due to age does not act in isolation but in conjunction with other factors, thus increasing the risk of vulnerability and discrimination. It is the case, for instance, of health, gender and economic inequalities associated to discrimination by reason of age.

As a conclusion we suggest a series of guidelines for public policies oriented to the reduction of above mentioned inequities 
que contribuya al desarrollo considerando las necesidades y potencialidades de cada ser humano.

PALABRAS CLAVE: Envejecimiento; Derechos Humanos; discriminación por edad; equidad; vulnerabilidad. and the construction of a fair society. A society that promotes development while taking into consideration the needs and potentialities of every human being.

KEY WORDS: Ageing; human rights; discrimination by age; equity; vulnerability. 


\section{Resumo}

\section{Saúde pública e os direitos dos idosos}

Levando-se que conta o não reconhecimento da comunidade internacional sobre os direitos dos anciãos, esse trabalho analisa a discriminação que em razão da idade sofrem os idosos.

São enfocadas as dimensões da saúde, profissional e intergeracionais com as três áreas onde são mais evidentes a discriminação. Distingue-se, outrossim, a vulnerabilidade relativa à idade e outros fatores, tais como, o gênero, a raça, a etnia ou o estrato sócioeconômico. A discriminação pela idade se expressa através de ações e omissões que impõem aos sujeitos padrões inferiores de atenção calcadas exclusivamente na maior idade. Fica evidente que a discriminação pela idade não age isoladamente, mas em conjunto com outros fatores que aumentam o risco de vulnerabilidade dos idosos. É o caso, por exemplo, da saúde, o gênero e as desigualdades sócioeconômicas.

Em conclusão sugere-se alguns procedimentos de políticas públicas orientadas a reduzir as iniquiidades apontadas na busca da construção de uma sociedade justa e desenvolvida considerando as necessidades e potenciais de cada ser humano.

PALAVRAS CHAVES: Envelhecimento; Direitos Humanos; discriminação por idade; equiidade; vulnerabilidade.

\section{Résumé}

\section{La santé publique et les droits des patients du troisème âge}

L'étude analyse la discrimination dont font l'objet les personnes âgées en fonction de leur âge, sachant que la protection de leurs droits n'a pas été reconnue par la communauté internationale comme un impératif.

Ces discriminations sont observées dans les domaines de la santé, du monde du travail et des transferts entre les générations. L'accent est mis sur la vulnérabilité en raison de l'âge, mais également pour d'autres facteurs, tels que le sexe, la race, l'ethnie, ou le statut socio-économique.

La discrimination par âge se manifeste à travers un ensemble d'actions ou d'omissions visant à considérer l'individu ou la collectivité avec des normes de qualité inférieure, en raison de leur âge uniquement. La discrimination par âge n'est pas un facteur isolé. ,elle agit en corollaire avec d'autres facteurs, augmentant ainsi le risque de vulnérabilité et accroissant la discrimination. C' est le cas par exemple de la santé, du sexe et des inégalités économiques, liées à la discrimination par âge.

En conclusion, l'auteur conseille de prendre toute une série de mesures de politique publique visant à réduire les inégalités en fonction de l'âge et à construire une société juste qui contribue au développement, en tenant compte des besoins et potentialités de chaque être humain.

MOTS CLÉS : vieillissement, droits de l'homme, discrimination par âge, équité, vulnérabilité. 


\section{Introducción}

Este documento aborda el tema de los derechos humanos de las personas mayores, entendiéndolo como parte de la reflexión general sobre los derechos humanos. La comunidad internacional reconoce dos categorías de derechos humanos: los derechos civiles y políticos y los derechos económicos, sociales y culturales (1). Estos derechos se derivan de supuestos básicos sobre el significado del ser humano y se aplican a todas las personas, independientemente de quiénes sean o dónde vivan. Junto a ello, la comunidad internacional ha reconocido explícitamente los derechos humanos de grupos vulnerables específicos, tales como las mujeres o los niños. Sin embargo, la necesidad de proteger los derechos humanos de los individuos en razón de la edad no ha recibido la misma atención.

La Declaración Universal de los Derechos Humanos, sancionada hace más de 50 años, reconoció el derecho a un nivel de vida adecuado y a contar con un seguro en situaciones de privación ajenas a la voluntad, entre ellas la vejez:

"Toda persona tiene derecho a un nivel de vida adecuado que le asegure, así como a su familia, la salud y el bienestar, y en especial la alimentación, el vestido, la vivienda, la asistencia médica y los servicios sociales necesarios; tiene asimismo derecho a los seguros en caso de desempleo, enfermedad, invalidez, viudez, vejez u otros casos de pérdida de sus medios de subsistencia por circunstancias independientes de su voluntad» (artículo 25, inciso 1).

El Pacto Internacional de Derechos Económicos, Sociales y Culturales de 1966 entrega el artículo más comprehensivo sobre el derecho a la salud en una ley internacional sobre derechos humanos. De acuerdo a este Pacto:

"Los Estados Partes en el presente Pacto reconocen el derecho de toda persona al disfrute del más alto nivel posible de salud física y mental" (artículo 12, inciso 1).

En ambos documentos no se reconocen explícitamente los derechos humanos de los adultos mayores, probablemente porque en el tiempo en que se redactaron el fenómeno del envejecimiento no había alcanzado las dimensiones actuales. Años más tarde, la Asamblea Mundial sobre Envejecimiento efectuada en Viena en 1982 aprobó el Plan de Acción Internacional sobre el Envejecimiento. Este el primer instrumento de política a nivel mundial para abordar las consecuencias y el impacto del envejecimiento en la vida de las personas y de las sociedades. El Plan establece 62 recomendaciones para adoptar medidas a nivel nacional que incorporen el envejecimiento en los planes y programas nacionales e internacionales de desarrollo.

El Plan de Acción no reconoce derechos humanos específicos de los adultos mayores. Sin embargo, reconoce el derecho a trabajar, a la jubilación y a la educación. El documento tampoco reconoce específicamente el derecho a la salud de los adultos mayores. No obstante, el ejercicio del derecho a la salud es interdependiente con muchos de los derechos sociales y económicos. Por ejemplo, no sólo la alimentación y el abrigo son determinantes sociales de la salud: la salud es, en muchos casos, una condición para que los individuos estén en capacidades de tener alimentos, nutrición y una vivienda segura. Una revisión de la Declaración Universal sobre los Derechos Humanos muestra que casi todos los derechos y libertades incluidos son a la vez componentes y determinantes de la salud.

En la primera parte de este documento abordamos la dimensión de salud, laboral y de las transferencias intergeneracionales de los adultos mayores en la Región como las tres áreas donde puede apreciarse la discriminación en razón de la edad, distinguiendo la vulnerabilidad de los adultos mayores en razón de su edad, de la vulnerabilidad de las personas debido a otros factores, tales como 
el género, la raza, la etnia o el estrato socioeconómico. En la segunda parte sugerimos políticas públicas para mejorar la situación de los mayores y promover una mayor inclusión social de este grupo etario.

\section{Diferenciación y discriminación por edad}

El envejecimiento es un proceso natural y la longevidad es un objetivo natural y deseable en cualquier sociedad. El envejecimiento es también un factor de riesgo para la disminución de la salud y de la funcionalidad. La acumulación de las características normales del envejecimiento define un umbral, el cual una vez traspasado, tiende a aumentar la propensión a la pérdida de las capacidades funcionales debido a la edad. Sin embargo, no toda la gente envejece de la misma manera. Está bien documentado que la calidad de vida y la funcionalidad en la vejez están relacionadas con las características personales y el estilo de vida, los recursos de la sociedad y el medio ambiente al que se está expuesto.

En la encuesta de Salud, Bienestar y Envejecimiento (SABE) en cinco ciudades de la Región, se aprecia que la mayoría de las personas de 60 años y más consideran que su salud no es buena y expresan no tener lo suficiente para satisfacer sus necesidades básicas del vivir diario. Si la utilización de la auto-percepción de la salud es un indicador significativo para medir la calidad de vida de los mayores, podríamos conjeturar que si los mayores en Bridgetown, La Habana, México, Santiago y Sao Paulo consideran que su salud no es buena, debemos indagar sobre otros indicadores que reflejen la iniquidad en salud en las personas mayores.

¿Es la auto-evaluación de salud una medida significativa para evaluar la calidad de vida de los mayores? A pesar de que el uso de evaluaciones de salud reportadas por cada individuo como una forma relativamente rápida, simple y de bajo costo de medir el es- tado de salud, es de bastante antigüedad, sólo recientemente se ha recopilado la evidencia suficiente para apoyar de manera convincente la idea de que las auto-evaluaciones de salud no son construcciones puramente imaginarias, no relacionadas a condiciones reales de salud. De hecho, en los últimos 15 años muchos estudios han demostrado que los individuos que reportan condiciones de salud escasa o pobre tienen riesgos de mortalidad consistentemente más altos que aquellos que reportan mejor salud. Esto ocurre aun después de controlar por un número de condiciones convencionalmente consideradas buenos predictores de la mortalidad. Por lo tanto, las auto-evaluaciones de salud son más que percepciones individualmente distorsionadas del estado de salud. Es más, se ha sugerido que la auto-evaluación del estado de salud es un buen indicador de propiedades o dimensiones latentes que son subyacentes a diferentes condiciones adversas de salud, tal como el estado físico y mental, así como un número de factores antecedentes del estado de salud, incluyendo depresión y estrés. La auto-evaluación de la salud también rescata la presencia de deterioros reales de salud que podrán estar o no bajo control o tratamiento. En conjunto, la relación entre la auto-evaluación y el conjunto de factores descritos anteriormente puede explicar por qué en una primera revisión de 27 estudios mundiales, se encontró que en 23 de ellos, la auto-evaluación de salud tuvo un efecto significativo sobre la mortalidad esperada, con los riesgos relativos asociados con la peor auto-evaluación relativa a la mejor cayendo dentro de un rango de 1,5-3,0. En un segundo análisis de 19 estudios publicados más recientemente, se encontró que la auto-evaluación de salud predecía bien la mortalidad en 17 de ellos, con los riesgos relativos cayendo dentro de un rango de 1,5 y 2,0.

En esta situación cabe preguntarse cuánto de la condición de los adultos mayores obedece a las condiciones de iniquidad de las sociedades, cuánto a hábitos pocos salu- 
dables y cuánto al hecho de que por su edad son discriminados o mal atendidos por los servicios de salud y excluidos de los beneficios económicos del mercado laboral. En otras palabras, nos preguntamos si las condiciones de salud y bienestar de los adultos mayores son el resultado de que están expuestos a prácticas discriminatorias o injustas en razón de su edad o en razón de condiciones que actúan independientemente de su edad. Evidentemente, la discriminación y las iniquidades por otros factores puede ser acentuadas por la edad, configurando una situación donde la edad y las condiciones sociales, económicas, culturales y políticas actúan sinérgicamente, impidiendo que los adultos mayores puedan hacer un ejercicio pleno de sus derechos humanos.

Para poder responder a la pregunta acerca de cuándo la edad es objeto de prácticas discriminatorias e injustas en una sociedad determinada, es necesario identificar cuáles son los indicadores que muestran prácticas inequitativas e injustas basadas en la edad.

Definimos equidad en su noción de justicia, diferenciándola del concepto de igualdad. La iniquidad alude a la existencia de desigualdades innecesarias, evitables e injustas, que se originan en condiciones sociales, económicas, culturales y ambientales diferenciales (2). La evitabilidad, la necesidad y, especialmente, la justicia, sitúan al concepto de equidad en una dimensión moral y ética, que puede llevar a definiciones distintas sobre lo que debiera considerarse como "equitativo" o, en otras palabras, "justo".

Definimos discriminación por edad, en distinción al concepto de diferenciación por edad, al conjunto de acciones u omisiones que tienden a tratar al individuo o a una colectividad con estándares inferiores en comparación con otros que no son miembros de esa colectividad definida en función de la edad.

¿Qué necesitaríamos observar en las per- sonas que han cumplido 60 años o más para decir que hay iniquidades y/o discriminación por edad que en diferentes casos se suma a otras iniquidades relativas al género y al nivel socioeconómico del individuo?

Necesitamos distinguir entre la discriminación que surge del efecto de la "cohorte", de aquella otra discriminación que puede estar relacionada a otros factores que las personas de 60 años y más comparten con otros grupos de la población. Adicionalmente, necesitamos distinguir las desigualdades presentes en la cohorte de mayores, que son al mismo tiempo innecesarias, evitables e injustas. Por ejemplo, el estilo de vida que los individuos adoptan a una edad temprana puede obedecer a distintas situaciones: una elección individual; una falta de información o de recursos; variaciones naturales o biológicas que no son causadas por acciones discriminatorias o injustas. Es decir, se trata de identificar, por una parte, cuáles son las desigualdades «evitables, innecesarias e injustas» en el acceso y la utilización de bienes y servicios esenciales para garantizar los derechos humanos básicos de los adultos mayores y, por otra, cuáles de estas desigualdades son producto de una discriminación implícita o explícita atribuibles a la edad.

Los indicadores más importantes para explorar el tema de la discriminación por edad son: el estado de salud, la participación en la fuerza de trabajo y las transferencias intergeneracionales. La dimensión de salud es una función de las condiciones que determinan el estado de salud de las personas mayores y el acceso y utilización de los servicios de salud. La dimensión laboral se refiere a la dinámica de la participación en la fuerza de trabajo de los adultos mayores, su edad y perfil educacional, como también su distribución por sector económico. La dimensión de las transferencias intergeneracionales se refiere a las transferencias institucionalizadas, tales como el sistema de pensiones, como también a los in- 
tercambios formales e informales entre los miembros de la familia.

Es posible ponderar estas dimensiones para producir indicadores integrados de la discriminación y la iniquidad relativa al envejecimiento en los países en desarrollo. Estos indicadores permitirían una evaluación formal de las medidas de protección que se necesitan para garantizar que los adultos mayores tengan un acceso justo a los bienes y servicios básicos, y para promover la asignación de recursos orientados a la protección de los derechos humanos básicos de los adultos mayores.

\section{La dimensión de salud}

A pesar de la importancia actual y futura que tiene la población de 60 y más años en los países de la Región, los actuales servicios de salud no tienen una política de reorientación de los servicios para la promoción, la prevención y la atención de salud de los mayores.

La Organización Mundial de la Salud (OMS) propone focalizar las intervenciones en salud pública durante el curso de la vida (figura 1) (3). Las intervenciones en los pri-

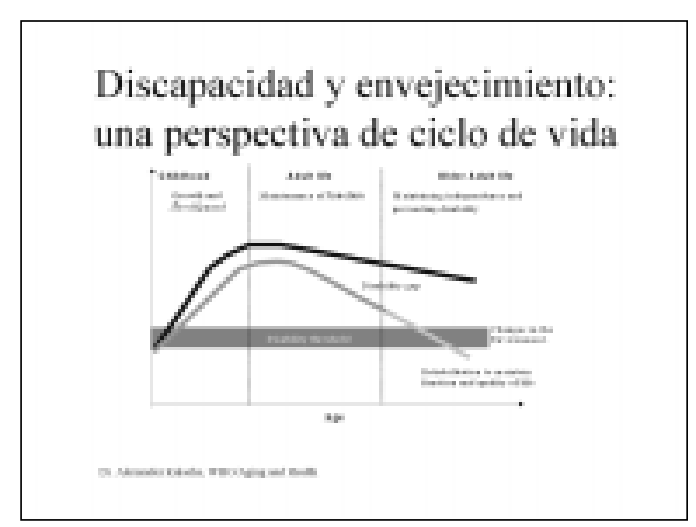

meros años de vida están destinadas a asegurar el desarrollo de la más alta capacidad funcional posible. Las intervenciones en la vida adulta están destinadas a retrasar la disminución de la funcionalidad. Para los adultos mayores que están por encima del umbral de la discapacidad, las intervenciones deben estar dirigidas a prevenir la pérdida de funcionalidad debido a causas evitables, como la malnutrición, caídas, enfermedades del corazón y reumatismo o artritis; enfermedades cerebrovasculares, diabetes, dificultades visuales, depresión, enfermedades malignas u osteoporosis. Finalmente, para quienes están por debajo del umbral de la discapacidad, las intervenciones deben estar dirigidas a reparar la pérdida de funcionalidad $\mathrm{y}$, cuando ya no es posible, asegurar la calidad de vida dentro de los límites impuestos por la discapacidad.

La mayor vulnerabilidad de los adultos mayores a los problemas de salud se traduce en que se requieren recursos humanos, tecnológicos y materiales diferenciados y apropiados para el cuidado de la salud, en una etapa de la vida en que las enfermedades tienden a ser crónicas y no agudas. La distribución de los recursos de salud y las barreras para acceder a ellos varían enormemente en los países de la Región. La decisión sobre la asignación de recursos de la salud pública para la prevención de la disfuncionalidad y la discapacidad es un importante tema de justicia distributiva relacionado con la salud pública y el envejecimiento.

Los siguientes indicadores pueden servir de guía para la identificación de aquellas situaciones en las cuales se nota negligencia política o social y que por consecuencia tienden a discriminar contra la salud de los adultos mayores.

\section{Indicadores de salud y bienestar}

La promoción de la salud en los adultos mayores empieza con el proceso de empoderamiento para: a) la participación activa en el auto-cuidado, b) la toma de decisiones sobre cambios de comportamiento, y c) demandar calidad de atención. Esencial para el empoderamiento de los mayores es que reciban información oportuna sobre prevención y manejo de factores de riesgo de enfermedades relacionadas con la edad, como información sobre el buen manejo de los medicamentos que está tomando. Una 
tercera parte de los adultos mayores de la Región no saben leer ni escribir y otra tercera parte tienen bajos niveles de educación. Por lo tanto, la educación en salud y la promoción de una cultura de auto-cuidado son elementos tan básicos para los adultos mayores como la alfabetización. Además, las siguientes preguntas son esenciales para empezar un análisis de la situación de los mayores y del nivel de inclusión que los mismos tienen en la agenda de salud pública del país:

- ¿Existe una campaña o programa de alfabetización en salud para los adultos mayores?

- ¿Existe malnutrición en los adultos mayores?

- ¿Llevan los adultos mayores vidas activas y participan en actividades físicas rigurosas?

- ¿Se sienten los adultos mayores satisfechos con la vida?

- ¿Cuál es la prevalencia de deterioro y discapacidad que no es justificable dentro de las expectativas de un envejecimiento digno y activo?

\section{Indicadores de calidad de atención}

Existe una relación epidemiológicamente demostrada entre la enfermedad y el envejecimiento. Esto no quiere decir que todas las enfermedades aumenten en función de la edad. Hay enfermedades que se relacionan con la edad, las cuales se asocian con más frecuencia a una edad específica y las enfermedades que dependen de la edad, por ejemplo la enfermedad de Alzheimer, la que aumenta su incidencia de forma exponencial en la medida que aumenta la edad. Los indicadores de calidad de atención para los adultos mayores deben demostrar el nivel de preparación que existe en una comunidad para prevenir y posponer las enfermedades que están más relacionadas con los adultos mayores, como aquellas que aumentan en función de la edad. Por lo tanto, se deben identificar los siguien- tes indicadores para medir el nivel de inclusión que tiene la salud de los adultos mayores en la programación de salud pública de una comunidad:

- ¿Está el personal de atención primaria capacitado para la promoción, prevención y atención a la salud de los adultos y las adultas mayores?

- ¿Se han adoptado guías y lineamientos de atención para posponer las discapacidades relacionadas con la edad en todos los niveles de atención?

- ¿Se incluye la participación de los adultos mayores en la toma de decisiones y en la evaluación de los servicios de salud?

- ¿Hay un presupuesto nacional y privado para invertir en tecnología de bajo costo y en medicamentos esenciales para el control de las enfermedades comunes en los adultos mayores?

- ¿Hay un presupuesto nacional y privado para investigaciones multidisciplinarias sobre el envejecimiento de la población?

La inversión necesaria para preparar al Estado y a la comunidad para el envejecimiento de la población es actualmente relativamente modesta si se considera que en los próximos 20 años esta población aumentará fuertemente en todos los países de la Región. Sin embargo, es necesario empezar con una inversión adecuada ahora, cuando todavía el porcentaje de personas mayores es relativamente bajo.

\section{Indicadores de acceso a los servicios de salud}

La privatización y la descentralización de los servicios de salud tienden a enfocar las prioridades de salud pública en base al costo-efectividad de sus acciones y no necesariamente con la justicia distributiva de los recursos de salud. La justicia distributiva exige que las personas reciban de acuerdo a sus necesidades y que las desigualdades fa- 
vorezcan a los más necesitados. ¿Cómo responde la política pública de salud a las necesidades de los adultos mayores con respecto a la cobertura y la accesibilidad de servicios apropiados de salud? Las siguientes preguntas permiten evaluar el nivel de acceso que tienen los mayores a una atención apropiada a los servicios de salud:

- ¿Discriminan los seguros de salud en contra de las personas mayores?

- ¿Hay acceso físico y económico a los servicios de promoción, prevención y atención a la salud incluyendo acceso a las tecnologías y medicamentos esenciales?

- ¿Hay acceso físico y económico a residencias de mayores y otros servicios comunitarios de atención a largo plazo?

- ¿El Estado se responsabiliza por registrar, reglamentar y monitorear los servicios y las viviendas de largo plazo para los adultos mayores discapacitados?

- ¿Existe una entidad gubernamental o nogubernamental que investiga y penaliza el abuso, maltrato y negligencia en contra de las personas mayores discapacitadas?

Si logramos utilizar no sólo indicadores de recursos sino también indicadores de impacto, estaríamos en mejores condiciones de documentar, entender y evaluar el rol de la salud pública y el envejecimiento en las sociedades en desarrollo. Más aún, se contaría con la capacidad para implementar estrategias prácticas destinadas a mejorar la calidad vida de los adultos mayores.

\section{La dimensión económica: participación en la fuerza de trabajo}

Los países de la Región están, en su conjunto, caracterizados por insuficientes tasas de crecimiento del producto interno bruto per cápita y elevados niveles de desempleo; parte importante del presupuesto nacional está al servicio de la deuda externa; hay un incremento en las disparidades de la distribución del bienestar y una disminución de nuevas inversiones en infraestructura y depreciación del capital. Todas estas condiciones macroeconómicas tienen importantes consecuencias en el nivel individual.

Gran parte de la población ha experimentado un decrecimiento sustancial en su ingreso real, una disminución de su acceso a los servicios de atención de salud y una disminución de su capacidad para demandar recursos públicos para la jubilación y la asistencia social (3). Los adultos mayores y los niños son los grupos más vulnerables a los impactos de estas tendencias.

La mayoría de los adultos mayores están inhabilitados para trabajar, ya sea por razones de edad o de discapacidad, y dependen del apoyo público como su fuente de ingreso, normalmente en la forma de beneficios gubernamentales provenientes de la seguridad social, subsidios, o ingresos monetarios provenientes de fondos de pensiones privados o semi-privados. Durante la última década, los adultos mayores han visto reducidos sus beneficios, ya sea por la vía de reajustes por debajo de la inflación o por la reducción de otros servicios.

Frente a estas tendencias, los adultos mayores tienen sólo dos posibles fuentes de ingreso. Una de ellas es el apoyo de las familias a través de compartir la vivienda o del intercambio regular de bienes y servicios. Esta opción es menos viable en la actualidad, debido a los cambios en la estructura de la familia, el incremento de la migración y la urbanización. La otra fuente de ingreso es continuar trabajando, ya sea en el sector formal o informal, sin posibilidades de jubilar y sin tener en cuenta el nivel de su capacidad funcional, el grado de estrés o el trabajar en ambientes poco saludables.

En resumen, el continuo deterioro del sistema público de pensiones (incluso cuando está disponible sólo para ciertos segmentos de la población) y de las tradicionales redes de apoyo familiar no ofrecerá a la mayoría de los adultos mayores la posibilidad de elegir la jubilación (4). Al mismo tiempo, otros 
factores como el deterioro de las condiciones de salud, la introducción de nuevas tecnologías y la falta de oportunidades para la capacitación y reactualización hacen más difícil la participación laboral. Es posible que la competencia por los distintos empleos, aun en el sector informal de la economía, dejará a muchos adultos mayores viviendo en la pobreza extrema durante los últimos años de su vida.

La relación entre la edad, la jubilación y la participación laboral es compleja. Sin embargo, la red de seguridad social debe ser lo suficientemente sólida como para asegurar que los adultos mayores no queden completamente desprotegidos en el momento en que la "edad" es en sí misma un factor de vulnerabilidad y pone en peligro el ejercicio del derecho a condiciones de vida que aseguren niveles básicos de bienestar y salud para sí mismos y para sus familias. En este sentido, la discriminación implícita o explícita en las prácticas de selección y contratación de personal en la gran mayoría de los países es un tema que requiere una urgente atención.

¿Qué sabemos acerca de los beneficios sociales que reciben los adultos mayores en América Latina? La asistencia social puede ser formal o informal. La disponibilidad de asistencia social formal para los adultos mayores pobres que no están en condiciones de trabajar podría darnos una medida de la "equidad" en relación a la edad y la discriminación laboral. La disponibilidad de intercambios familiares y del apoyo social a los cuidadores familiares de personas mayores medirá el nivel de "equidad" que existe entre las generaciones.

La población de adultos mayores que vive en condiciones de pobreza seguirá creciendo (5). Como el número de adultos mayores se incrementa, los costos para protegerlos también se incrementan. A menos que la sociedad se organice y desarrolle políticas orientadas a los adultos mayores, la combinación de envejecimiento con el incremento de la pobreza podría convertirse en un grave problema que afecte a los individuos como a la sociedad en su conjunto.

\section{Indicadores de equidad social y económica}

La pobreza y el género, conjuntamente con la edad, tienden a crear una situación de negligencia y abandono social para las personas mayores, principalmente las mujeres mayores. Esta situación se hace invisible por el estereotipo que presenta la vejez como regreso a la dependencia y a la marginalización.

- ¿Existe un consenso nacional para diseñar los mecanismos centrales que puedan garantizar los derechos humanos durante la vejez?

- ¿Existe legislación prohibiendo la discriminación por edad en el trabajo?

- ¿Tienen los retiros y las pensiones sociales una cobertura amplia que responda a las necesidades reales de protección social requerida por personas de mayor edad?

- ¿Discriminan por edad los préstamos de desarrollo social y micro-empresas?

- ¿Está el transporte público adaptado a las necesidades de las personas con discapacidad y/o fragilidad?

\section{La dimensión de las transferencias intergeneracionales}

En la mayoría de los países en desarrollo, la manutención de los adultos mayores es en gran parte realizada por intercambios en los que participa la familia ${ }^{i}$. Estos intercambios no son siempre unidireccionales, ya que los adultos mayores saludables, especialmente las mujeres, reciben bienes y servicios a cambio de un conjunto de bienes y

Hermain AI, Ofstedal MB, L. Chi.. Kin Availability of the Elderly in Taiwan: Who is available and where are they?. Comparative Study of the Elderly. Asia Research Reports 1989;(5) 9218. University of Michigan, Population Studies Center, en: Palloni, A. And Devos, S.. "Formal Models and Methods for Analyzing Kinship and Houselhold Organization. Population Index 1989; 55(2). 
servicios que ellos mismos proveen al hogar. En las familias pobres, el hogar de las personas más viejas de la familia se convierte en el hogar de los hijos y los nietos y la abuela continúa atendiendo las necesidades de la familia. A cambio, el trabajo de los miembros de la familia permite tener un ingreso para asegurar las mínimas condiciones de vida. Este escenario es posible por la acción de dos factores: la disponibilidad de los miembros de la familia y su capacidad y buena voluntad para ser parte de este intercambio; y la buena voluntad y capacidad de los adultos mayores para ser parte del mismo intercambio. Cuando un adulto mayor ya no está en condiciones de contribuir, es frecuente que el intercambio pierda viabilidad.

En orden a evaluar la situación de las transferencias intergeneracionales familiares y las condiciones bajo las cuales son viables, necesitamos observar los arreglos familiares a través de un período de tiempo, de modo tal que nos permita identificar los efectos de la edad y la salud en esta relación. Un estudio sobre los arreglos familiares de los adultos mayores en América Latina utilizando los Censos de Población mostró una tendencia al aumento en la proporción de adultos mayores hombres y mujeres que viven solos (6). Esto no es necesariamente un indicador del deterioro de los intercambios familiares y necesitamos muchos más datos para aprender a medir la forma en que los adultos mayores están sobreviviendo a pesar de la pobreza.

Frente a la inexistencia de una red de protección social para los adultos mayores, es posible que la red familiar se convierta en el único medio de sustento para los adultos mayores que se han vuelto vulnerables a causa de la edad, la salud y la pobreza. Las políticas públicas orientadas a definir la responsabilidad social y familiar en una sociedad en envejecimiento son esenciales para preservar los derechos humanos de los adultos mayores. Postulados éticos relacionados con la justicia y la equidad sugieren que las familias pobres no debieran tener la responsa- bilidad total de la protección de los adultos mayores a expensas de la manutención de los miembros jóvenes de la familia. Por lo tanto, los siguientes indicadores miden el nivel de inclusión de los mayores en las políticas sociales del país:

\section{Indicadores de protección social y familiar}

- ¿Existen opciones de apoyo y servicios para las familias de los adultos mayores con discapacidad?

- ¿Existen opciones de viviendas y de servicios diurnos para los adultos mayores sin red familiar?

- ¿Los adultos mayores están protegidos por la ley contra el abuso, maltrato y negligencia?

- ¿Los adultos mayores, por el hecho de tener una discapacidad, mantienen el derecho de tomar decisiones?

En resumen, nos hemos preguntado en este artículo si los adultos mayores son vulnerables al abuso, la discriminación y a una existencia marginal por razón de su edad o si ellos son discriminados porque son pobres. Hemos tratado de mostrar cómo, en la población en los países en desarrollo, la vejez, la salud, la pobreza y las iniquidades aparecen íntimamente relacionadas.

La vulnerabilidad se define como la falta de capacidades de un individuo o un grupo para satisfacer la mayoría de sus necesidades básicas: salud, educación y nutrición, vivienda y educación. Por ejemplo, una viuda de 65 años que sufre una discapacidad y que no tiene una pensión es vulnerable porque se encuentra con una serie de dificultades para satisfacer los requerimientos mínimos para su vida. Adicionalmente, ella encontrará que no tiene acceso a la educación, empleo, fondos de desarrollo comunitario y cuidado de la salud en razón de su edad. Esto la hace doblemente vulnerable. También, como mujer, ella se encontrará vulnerable al abuso y la violencia en el hogar. La socie- 
dad no tiene un espacio para las mujeres viejas y discapacitadas, que ya no son productivas y que, por el contrario, compiten con otros en la familia por los limitados recursos familiares y sociales. Esencialmente, estamos hablando de un triple riesgo o vulnerabilidad: la salud, el género y las desigualdades económicas asociadas a la discriminación por edad.

La situación de discriminación y vulnerabilidad en que vive gran parte de los adultos mayores de la Región les niega el ejercicio de sus derechos humanos básicos, consagrados en distintos documentos y tratados internacionales.

¿Qué clase de políticas públicas deben formular los gobiernos para reducir las iniquidades relacionadas con la edad? Las opciones deben incluir políticas orientadas a brindar a cada persona las oportunidades para su propio desarrollo, tales como invertir en aprendizaje durante toda la vida, una vivienda adecuada, un sistema integrado de cuidados de la salud en el nivel primario, y un empleo digno. Además, una sociedad justa debiera formular políticas orientadas a entregar, a cada persona, protección para los tiempos de enfermedad, desempleo, viudez o abandono. Estas políticas no sólo se justifican desde el principio del bienestar individual, sino también desde el principio de la justicia distributiva.

Para abordar en forma comprehensiva el problema del ejercicio de los derechos humanos de los adultos mayores, los gobiernos deberían comenzar por enfrentar el tema de la salud, el género y las desigualdades económicas directamente relacionadas a la edad. La participación de los adultos mayores en los procesos políticos y su nivel de información y de movilización serán determinantes en la reacción de los gobiernos locales frente a estos temas.

El envejecimiento de la población, la salud y la pobreza tienen implicancias para el desarrollo y pueden convertirse en un obstá- culo si no se toman oportunamente las medidas adecuadas. Por lo tanto, el desarrollo de una infraestructura justa y sustentable para el envejecimiento de la población tendría que formar parte de la agenda internacional del desarrollo.

En una sociedad justa, todas las personas - jóvenes y viejas - disfrutarán la clase de oportunidades y protección que cada uno necesita para mantener la salud, la dignidad y el bienestar a lo largo del ciclo de vida. En una sociedad justa, la carga del desarrollo es distribuida de acuerdo a las fortalezas y capacidades y la red de protección es distribuida de acuerdo a las discapacidades y necesidades. Una sociedad justa es una sociedad que contribuye al desarrollo sin consideración de la edad, el género y el origen étnico, sino en consideración a lo que cada ser humano necesita.

En la Segunda Asamblea sobre Envejecimiento que se realizará en abril del año 2002 la discusión se centrará en una visión y elaboración de un plan para el envejecimiento en el siglo XXI. Esperamos que la comunidad internacional defina una agenda adecuada para abordar los cambios en el envejecimiento en los países en desarrollo y para asegurar el ejercicio pleno de los derechos humanos de los adultos mayores.

\section{Referencias}

1. Mann JM, Gruskin S, Grodin MA, Annas GJ. Health and human rights. New York: Routledge; 1999.

2. Whitehead M. Los conceptos y principios de la equidad en salud. Washington, D.C: OPS/ OMS. Centro de Documentación e Información CID, Programa de Desarrollo de Políticas de Salud (HSP); 1991.

3. WHO. Ageing and Health. A life course perspective of maintaining independence in older age. Geneva: WHO/HSC/AHE/; 1999.

4. Veira-Ramos A. Assesing the influence of health status: financial resources and job displacement of early retirement. Thesis. Madison: Center for Demografy \& Ecology, 
University of Wisconsin; 1998.

5. Mesa Lago C. Old-age security and helath care for the poor in Latin American and the Caribbean. Paper presented at the Conference on Social Protection and Poverty, Inter American Development Bank. 1999.
6. Palloni A, DeVos S. Aging and Latin America and the Caribbean. Madison: Center for Demography and Ecology. University of Wisconsin, CDE Working Paper No.99-02; 1989. 